\title{
Expressions for Precession Formulae
}

\author{
P. Bretagnon \\ Bureau des Longitudes, Paris, France
}

In 1976 , the LAU recommended for the value of the precession constant $50^{\prime \prime} 290966$ per Julian year and, since 1984, most of the ephemerides have used the formulas of Lieske et al. (1977). In this paper, the motion of the ecliptic was based on Newcomb's theory. The ecliptic was defined with an accuracy of $0^{\prime \prime} 3$ over 1000 years and of 6 mas over one century around J2000. For several years, new determinations of the precession constant have showed that this value is too high by about 3 mas per year. For instance, Williams et al. (1991) give 50"2882 per year. From this value and from the mean elements defining the ecliptic given by Simon et al (1994), we have built new expressions for precession formulae. The motion of the mean ecliptic is described through variables $q$ and $p$ :

$$
\begin{aligned}
& q \times 10^{10}=-11346887 t+123731 t^{2}+12654 t^{3}-137 t^{4}-3 t^{5}, \\
& p \times 10^{10}=1018038 t+470200 t^{2}-5417 t^{3}-251 t^{4}+5 t^{5},
\end{aligned}
$$

where $t$ is the time reckoned in thousands Julian years from J2000. This solution has an accuracy of 1 mas over several thousands years and 0.1 mas over one century around J2000. The variations of the equator are obtained by integration of the differential equations of the precession in longitude and of the obliquity in Kinoshita (1977) and Kinoshita and Souchay (1990). The dynamical ellipticity is determined in order to obtain $(d \mathcal{P} / d t)=50288^{\prime \prime} 2$ at $t=0$ as $H_{d}=0.0032737752$. From this value for $H_{d}$, we can integrate the differential equations for $\mathcal{P}$ and $\varepsilon$ :

$$
\begin{aligned}
& \mathcal{P}=50288^{\prime \prime} .200 t+111^{\prime \prime} .2022 t^{2}+0^{\prime \prime} .0773 t^{3}-0^{\prime \prime} .2353 t^{4}-0^{\prime \prime} .0018 t^{5}+0^{\prime \prime} .0002 t^{6}, \\
& \varepsilon_{0}=23^{\circ} 26^{\prime} 21^{\prime \prime} .412-468^{\prime \prime} .0927 t-0^{\prime \prime} .0152 t^{2}+1^{\prime \prime} .9989 t^{3}-0^{\prime \prime} .0051 t^{4}-0^{\prime \prime} .0025 t^{5} .
\end{aligned}
$$

Formulae (1) and (2) enable one to compute all the precessional quantities. These quantities were computed using the IAU 1976 planetary masses. We have better determinations now of these masses (McCarthy, 1992, for example). Taking into account the order of magnitude of the uncertainties on the masses, we have computed the derivatives with respect to the masses of Mercury through Neptune. The error on the Earth-Moon mass is too small to have an influence. Likewise, we consider that the uncertainty on the precession constant is smaller than $0^{\prime \prime} .5$ per thousand Julian years; those on $\varepsilon_{0}$ is smaller than $0^{\prime \prime} .1$ and we have computed the derivatives of the precessional quantities with respect to the precession constant and with respect to the obliquity. We have therefore established the derivatives with respect to all the physical constants occuring in the precessional quantities. These derivatives will be useful for the further improvements of the planetary masses, the precession constant and the obliquity. To improve the precessional quantities, it will be good to proceed to a systematic treatment of the second-order effects taking into account, for instance, some lunar perturbations due to the motion of the ecliptic (Williams 1994). But these effects are too small to modify the derivatives of the precessional quantities. The formulae of the precessional quantities and of the derivatives are given in Simon et al (1994) and are available by anonymous FTP in the CDS of Strasbourg.

\section{References}

Kinoshita H., 1977, Celes. Mech. 15, 277.

Kinoshita H., Souchay J., 1990, Celes. Mech. 48, 187.

Lieske J. H., Lederle T., Fricke W., Morando B., 1977, A\&A 58, 1.

McCarthy D. D., 1992, IERS Standards, IERS Technical Note 13, Observatoire de Paris.

Simon J. L., Bretagnon P., Chapront J., Chapront-Touzé M., Francou G., Laskar J, 1994, A\&A 282, 663.

Williams J. G., Newhall X X, Dickey J. O., 1991, A\&A 241, L9.

Williams J. G., 1994, AJ 108, 711. 